\title{
MULTISPECTRAL ANALYSIS OF IRAS SKY FLUX MAPS
}

\author{
L. G. Balázs and M. Kun \\ Konkoly Observatory \\ Box 67, 1525 \\ Budapest, Hungary
}

\author{
V. Tóth \\ Eötvös University \\ VIII. Kun B. ter 2 \\ Budapest, Hungary
}

\begin{abstract}
We have tested the performance of principal components analysis and a single-pass clustering algorithm to identify different components of the cosmic dust. Applying these techniques on a training set of 2500 points extracted from the PL51 IRAS maps we recognized two main components with temperatures of $180 \mathrm{~K}$ and $28 \mathrm{~K}$.
\end{abstract}

\section{INTRODUCTION}

Even the first results of the IRAS mission (Hauser et al. 1984; Low et al. 1984) revealed a variety of components of different temperatures. The maps of the extended sources are the result of the superposition of radiation coming from these different components, i.e., in the optically thin case we get

$$
F(\lambda)=\sum c_{j} B\left(l, T_{j}\right) \quad \lambda=12,25,60,100 \mu
$$

where $F(\lambda), c_{j}, B$, and $T_{j}$ are the measured flux, the column density, the emissivity, and the temperature of the dust particles, respectively.

\section{PRINCIPAL COMPONENTS ANALYSIS}

The 12, 25, 60 and $100 \mu$ colors of each pixel of the IRAS maps define a point in a fourdimensional parameter space. As an example of our multispectral studies we investigated the IRAS field PL51 $\left(\mathrm{RA}=4 \mathrm{~h}\right.$; $\left.\operatorname{dec}=+30^{\circ}\right)$ in more detail. We have extracted by random sampling a training set of 2500 points from the original Sky Flux Maps. Principal components analysis separates the observed data into the linear combinations of the eigen vectors of the correlation matrix of the data. (For further details see Murtagh and Heck 1987.) Factor analysis keeps only those eigen vectors which have eigen values above a certain threshold. Table 1 summarizes the results. The first factor almost fully explains the $25 \mu$ flux which is heavily dominated by the Zodiacal Light. The second factor, in contrast, describes the effect of galactic dust which produces most of the $100 \mu$ emission.

TABLE 1A

\begin{tabular}{cc}
\hline \hline eigen value & cum. percent. \\
\hline 2.4818 & 62.0 \\
1.3910 & 96.8 \\
.1003 & 99.3 \\
.0268 & 100.0 \\
\hline
\end{tabular}

TABLE 1B

\begin{tabular}{lrr}
\hline \hline variable & 1.factor & 2.factor \\
\hline F12 & .9637 & .2089 \\
F25 & .9917 & .0458 \\
F60 & .3625 & .9044 \\
F100 & -.0409 & .9819 \\
\hline
\end{tabular}




\section{CLUSTER ANALYSIS}

The $c_{j}$ column densities may have some grouping tendencies in color space. We utilize a commonly used method, a single pass clustering algorithm (see Murtagh and Heck 1987). We set $k=10$ for the number of clusters and performed the algorithm in the space defined by the factors obtained in the previous paragraph. The clusters localized by this algorithm are displayed in a factor-factor plot shown in Figure 1. The basic features on this plot are the two "fingers" pointing upwards and nearly horizontally. These "fingers" may be identified with the effect of Zodiacal Light (dominating $f 1$ ) and the galactic radiation (dominating $f 2$ ). To obtain information on the temperatures of these components we divided the fluxes at 12,25 , 60 , and $100 \mu$ by $F=F 12+F 25+F 60+F 100$. Figure 2 displays the $F 25 / F$ by $F 60 / F$ plot where the plotted cluster members are coded by the serial number of their clusters. The wedge-like distribution of the plotted cluster symbols points to the $180 \mathrm{~K}$ and $28 \mathrm{~K}$ values on the dust law $\alpha=1$ radiation curve (Leene 1988) suggesting that a mixture of these radiations is responsible for the $12,25,60$, and $100 \mu$ maps of PL51.

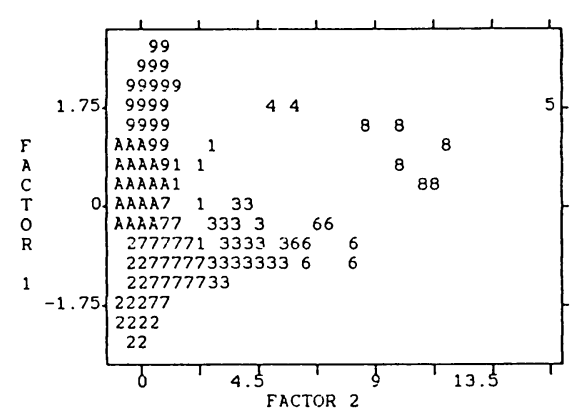

Figure 1. Plot of clusters on the factor plane found by the clustering algorithm. The cluster members are coded by the serial number of the respective cluster. (A denotes cluster 10.)

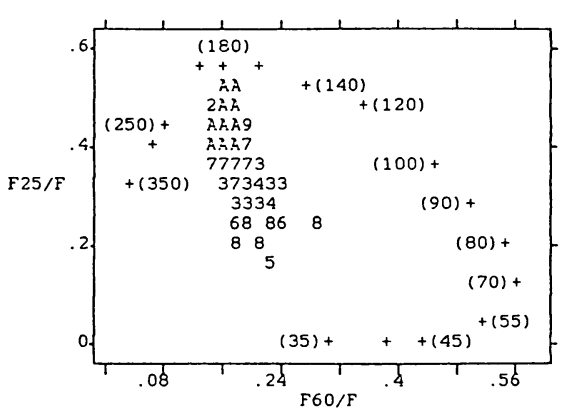

Figure 2. Distribution of cluster members on the $[F 25 / F ; F 60 / F]$ plane. The coding of clusters is the same as on Figure 1. The loci of dust law $\alpha=1$ radiation of different temperatures are marked with crosses. The numbers in parentheses are the respective temperatures. Note that the wedge-shaped distribution of symbols points towards the $28 \mathrm{~K}$ and $180 \mathrm{~K}$ temperatures.

\section{REFERENCES}

Hauser, M. G. et al. 1984, Ap. J., 278, L15

Leene, A. 1988, “IRAS Studies of the Nature of Interstellar Dust and Planetary Nebulae," Ph. D. thesis. Low, F. J. et al. 1984, Ap. J., 278, L23.

Murtagh, F., and Heck, A. 1987, Multivariate Data Analysis (Dordrecht: Reidel). 